\title{
Rapidly grown KDP crystals
}

\author{
V.I. Salo \\ STC "Institute for Single Crystals", Department of Opt. and Constr. Crystals; \\ Lenin Ave. 60; 310001 Kharkiv, Ukraine; \\ Phone: 380(572)308307; Fax: 380(572) 320019; e-mail: salo@isc.kharkov.com
}

\begin{abstract}
Results of the study of optical characteristics, real structure and laser damage threshold of KDP crystals rapidly grown in the direction of a prespecified angle of synchronism $(\theta=$ $59^{\circ}$ ) are described in the present paper. A comparative analysis of KDP characteristics for crystals grown by conventional method at a rate of $1 \mathrm{~mm} /$ day and by the method of oriented growth at a rate of $10 \mathrm{~mm} /$ day is presented. It is shown that elaborated method allows to obtain blanks of nonlinear elements already at the stage of growth. The grown crystals have the bulk laser damage threshold on the level of $\sim 5 \cdot 10^{10} \mathrm{~cm}^{2}$ and high structure quality; thus it allows to apply them as multipliers of laser radiation frequency.
\end{abstract}

Keywords: KDP, single crystals, real structure, crystallization parameters, optical absorption, laser damage threshold.

Paper received 19.09.99; revised manuscript received 13.11.99; accepted for publication 21.03.00.

\section{Introduction}

Creation of megajoule laser systems raised a problem of operative furnishing of these systems with frequency multipliers with the aperture of $1 \mathrm{~m}$. The problem of producing such elements cannot be solved in the limits of conventional method [1-4].

The present paper is devoted to rapid growth of large $\left(200 \times 200 \mathrm{~mm}^{2}\right.$ in cross - section) KDP crystals at the angle of synchronism $\left(\theta=59^{\circ}\right)$ and to investigation of their properties.

The method of such oriented growth consists of the following: growth of crystals proceeds in usual single chamber crystallizers by the method of temperature lowering. Realization of the new method became possible owing to the successful solution of the following problems:

1) the problem of solution stability at high supersaturation;

2) elaborating of a device directing the growth of crystals at the angle of $\theta=59^{\circ}$;

3 ) working out the growth regimes by the selection of optimal hydrodynamic conditions.

\section{Experiment}

Taken for the study were KDP crystals, $200 \times 200 \mathrm{~mm}^{2}$ in cross - section, grown by:

1) the traditional method on a seed in the form of $Z$-cut in the direction [001] at a rate of $1 \mathrm{~mm} / \mathrm{day}$;

2) the method of oriented growth at a rate of $10 \mathrm{~mm} /$ day.

In both cases the crystals were grown from the raw material of equal degree of purity by lowering the temperature. Samples $20 \times 20 \times 20 \mathrm{~mm}^{3}$ in size were cut from the grown crystals; further they were optically treated.

\section{Results}

\subsection{Real structure of KDP crystals}

The study of structural quality of KDP crystals was carried out by the multicrystal $\mathrm{X}$ - ray diffraction analysis of high resolution.

Comparative study of structural quality of KDP(1) crystals (grown by the conventional method) and KDP (2) ones (grown by the authors' elaborated method) are given in Table 1. 


\section{V.I. Salo: Rapidly grown KDP crystals}

Table 1.

\begin{tabular}{|c|c|c|c|c|c|c|c|c|c|c|}
\hline \multirow[t]{2}{*}{ Sample } & \multicolumn{3}{|c|}{$\beta$, arcsec } & \multicolumn{2}{|r|}{$\sigma_{\beta}$} & \multicolumn{2}{|c|}{$I^{R} 10^{-6} \mathrm{rad}$} & \multicolumn{3}{|c|}{$\sigma_{I}^{R}$} \\
\hline & $(008)$ & (206) & $(026)$ & $(008)$ & $(206)(026)$ & $(008)(206)$ & $(026)$ & (008) & $(206)$ & (026) \\
\hline KDP(1) & 7.69 & 6.53 & 6.52 & 0.28 & $0.66 \quad 0.65$ & 8.891 .59 & 1.58 & 0.19 & 0.19 & 0.19 \\
\hline $\mathrm{KDP}(2)$ & 6.84 & 4.76 & 5.40 & 0.32 & $0.94 \quad 0.98$ & $8.38 \quad 0.92$ & 0.98 & 0.34 & 0.34 & 0.32 \\
\hline
\end{tabular}

As one can see from Table 1 , the value $\bar{\beta}, \bar{I}^{R}$ for the studied samples of KDP (2) is lower than for KDP(1) crystals, which testifies to a high structure perfection as to linear defects in the crystals under investigation. However, one must not exclude the presence of micropores and microinclusions that do not influence significantly on $\bar{\beta}$ and $\bar{I}^{R}$ but can produce an effect on $B L D T$. The analysis of diffraction reflection curve $(D R C)$ for $\mathrm{KDP}(2)$ samples showed that typical for them is break of the curve into 4-5 maxima, angular distances between which are $\sim 0.5$ arcs. Taking into account that the cross-section of the incident X-ray beam for the reflection $\{206\},\{026\}$ is $73-75 \mathrm{~mm}$ and the presence of 4-5 separated maxima on DRC is easy to determine the cross - section of the crystal areas that give such turns. It makes 15-20 $\mu \mathrm{m}$.

For $\mathrm{KDP}(1)$ samples break of DRC is observed rarely, and turn angles were $1-3$ arcs; the number of these maxima was as a rule 2-3. A peculiar feature of the measured parameters for $\mathrm{KDP}(2)$ were almost two times higher values of dispersion $\sigma_{\beta}, \sigma_{I}^{R}$ and crystallographic anisotropy of $\bar{\beta}, \bar{I}^{R}$ parameters.

The origin of low angle turns is explained by the mechanisms of layer-by-layer growth, nonuniform entry and distribution of impurities, microdefects in the volume of the grown crystal.

\subsection{Light absorption}

KDP crystals are practically transparent in the visible and near IR parts of the spectrum. However, crystals grown on point seeds have, as a rule, the increased absorption in the growth sectors having enormous area of the volume which are formed by the facets of different indices.

Shown in Fig. 1 are the absorption spectra of traditionally grown crystals (1) and of those grown with orientation (2).

The absorption coefficient for rapidly grown crystals at the wavelength of the fourth harmonics $(\lambda=266 \mathrm{~nm})$ has the value $\sim 0.03 \div 0.04 \mathrm{~cm}^{-1}$ (for traditionally grown crystals it is $0.02 \div 0.03 \mathrm{~cm}^{-1}$ ).

The obtained results testify to the fact that growth of crystals in the direction of the prespecified angle of synchronism does not make worse the transparency. Moreo-

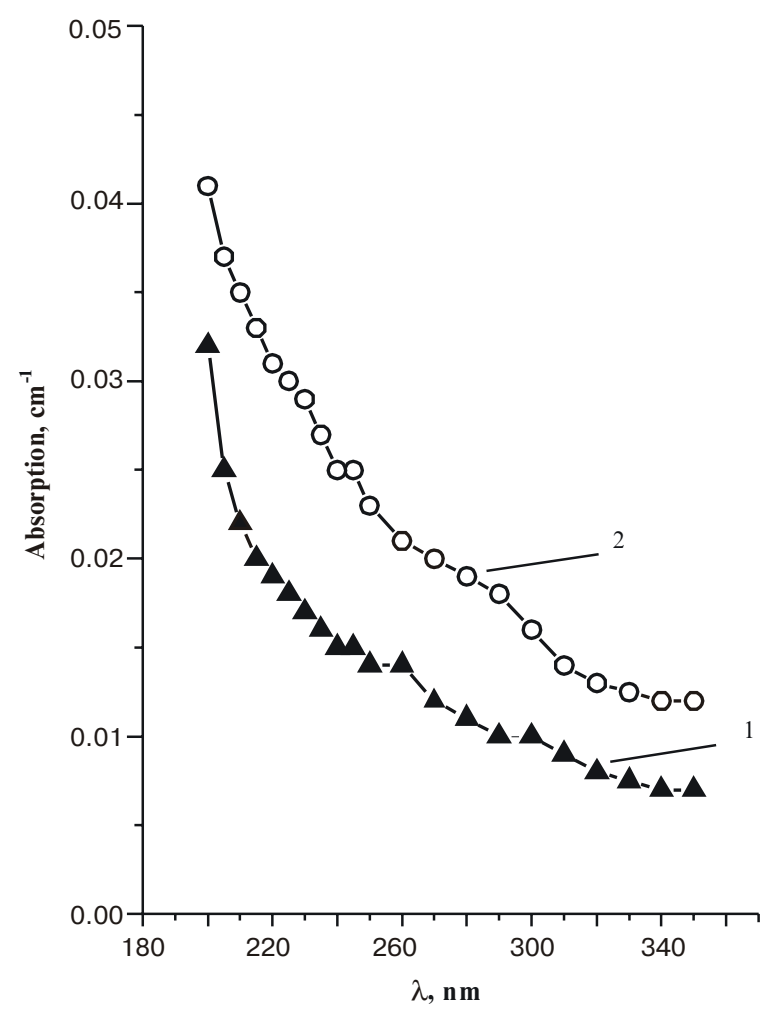

Fig. 1. Absorption spectra of traditionally grown crystals (1) and of those grown with orientation (2).

ver, there is no absorption in the growth sectors formed by the facets $\{100\}$. Thus, from the point of view of UV absorption crystals grown at an angle of $\theta=59^{\circ}$ can be used for efficient conversion of radiation of YAG: $\mathrm{Nd}^{3+}$ laser $(\lambda=1064 \mathrm{~nm})$ into the second $(\lambda=532 \mathrm{~nm})$ and the third $(\lambda=353 \mathrm{~nm})$ harmonics.

\subsection{Laser damage threshold measurements}

A schematic diagram of the experimental arrangement for laser damage studies is shown in Fig. 2.

The results of the measurements of bulk laser damage threshold of $\operatorname{KDP}(1)$ and $\operatorname{KDP}(2)$ crystals are shown in Table 2. 


\section{V.I. Salo: Rapidly grown KDP crystals}

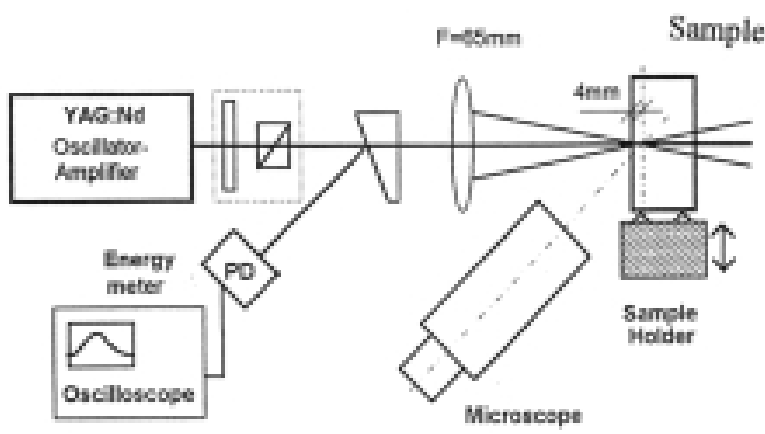

Fig. 2. Laser damage experimental set up. Laser radiation parameters:

Wavelength - $1064 \mathrm{~nm}$;

Pulse width: $14 \mathrm{~ns}$;

Intensity spatial distribution: $\mathrm{I}=\mathrm{I}_{\mathrm{o}} \exp \left(-\mathrm{R}^{2} / \mathrm{R}_{\mathrm{o}}{ }^{2}\right)$;

Beam waist diameter $\left(\mathrm{FW} \mathrm{1/eM):} 2 \mathrm{R}_{\mathrm{o}}=20\right.$ microns.

Table 2.

\begin{tabular}{|c|c|c|}
\hline \multirow[t]{2}{*}{ Sample } & \multicolumn{2}{|c|}{$\begin{array}{l}\text { Laser Damage Threshold } \\
1011 \mathrm{~W} / \mathrm{cm}^{2}\end{array}$} \\
\hline & $\min$ & $\max$ \\
\hline$\overline{\mathrm{KDP}}(1)$ & 4.8 & 5.5 \\
\hline KDP(1) & 0.5 & 0.7 \\
\hline $\mathrm{KDP}(2)$ & 0.3 & 0.4 \\
\hline $\mathrm{KDP}(2)$ & 0.5 & 0.7 \\
\hline
\end{tabular}

The presented results demonstrate that the value of bulk laser damage threshold of KDP crystals grown at the angle of synchronism $\theta=59^{\circ}$ is on the level of that for traditionally grown crystals.

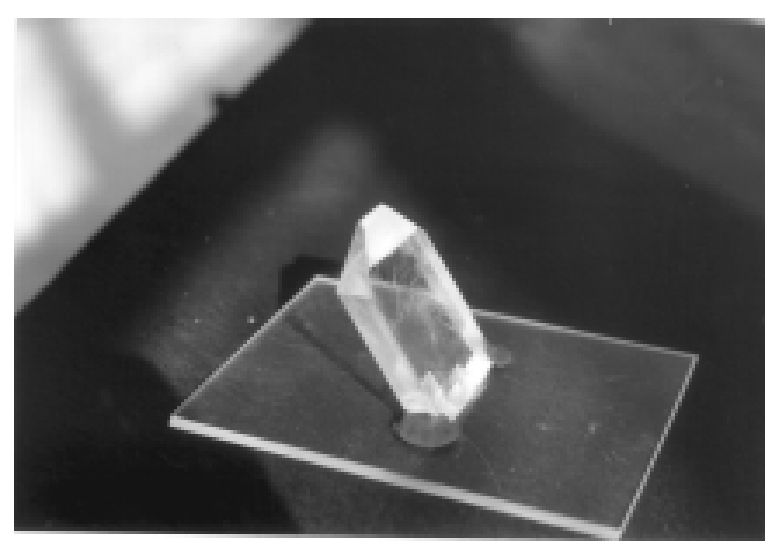

Fig. 3. Rapidly grown orientated $\left(\theta=59^{\circ}\right)$ KDP single crystals.

\section{Conclusions}

The carried out complex investigations of KDP crystals grown at the angle of synchronism $\theta=59^{\circ}$ (Fig. 3) allow to make a conclusion that the elaborated technology gives a possibility to obtain (directly in the growth process) blanks of nonlinear elements with the parameters that meet the requirements of laser engineering.

\section{References}

1. L. N. Rashkovich, KDP - family single crystals, IOP, Bristol, (1991).

2. A. A. Chernov, N. P. Zaitseva and L. N. Rashkovich, Rapid growth of KDP crystals // J. Crystal Growth, 102, pp. 793 796 (1990).

3. K. Fujioka, S. Matsuo, T. Kanabe, H. Fujita, and M. Nakatsuka, Optical properties of rapidly grown KDP crystals improved by thermal conditioning // J. Crystal Growth, 181, pp. 265 - 271 (1997).

4. M. Nakatsuka, K. Fujioka, T. Kanabe, H. Fujita, Rapid growth over $50 \mathrm{~mm}$ /day of water-soluble KDP crystals // $J$. Crystal Growth, 171, pp. 531 - 537 (1997). 